\title{
Location information-assisted opportunistic beamforming in LTE system for high-speed railway
}

\author{
Meng Cheng ${ }^{*}$ and Xuming Fang
}

\begin{abstract}
Communication systems of high-speed railway have inherent disadvantages and advantages. The estimation error of direction-of-arrival (DOA) of desired users caused by channel feedback delay and high moving speed make the common beamforming technique of smart antennas work abnormally. Instead, the opportunistic beamforming (OBF) with dumb antennas which does not need channel feedback is proper for high-speed railway. As the system has linear topology, regular movement as well as predictable location and speed information, the OBF can be improved to adapt to this scenario. In this article, conventional OBF is first introduced to the communication systems of high-speed railway. By multiplying random complex on each transmit antenna, the channel fluctuation dynamic range is extended so that multiuser diversity can be exploited. And then location information is employed to assist the conventional one. Communication systems in high-speed railway have the advantage of predictable location and speed information. The improved OBF can perform closer to the performance of coherent beamforming. Numerical analysis and simulation results show that both two algorithms can improve the system performance significantly.
\end{abstract}

\section{Introduction}

As the industry of high-speed railway develops very fast, people pay a lot of attention to the reliability and efficiency of communication systems for high-speed railway. High mobility of onboard mobile stations brings about Doppler Effect and frequent handovers which are sources of hindrance to high performance communications. However, it has some special characteristics such as linear topology of cells, so the movement is regular and location as well as speed information is predictable [1]. If these features are properly made use of, they can compensate for the disadvantages to some extent, even bring about some gains.

Several advanced and novel technologies of LTE can be applied to communications for high-speed railway, since GSM for Railway will evolve to LTE for Railway (LTE-R) [2]. Multi-antenna and smart antenna techniques are widely used to solve the problems existing in high-speed railway [3]. Since most parts of the carriage

\footnotetext{
* Correspondence: joycecheng.meng@gmail.com

Institute of Mobile Communications, Southwest Jiaotong University, Chengdu 610031, China
}

\section{Springer}

(c) 2012 Cheng and Fang; licensee Springer. This is an Open Access article distributed under the terms of the Creative Commons Attribution License (http://creativecommons.org/licenses/by/2.0), which permits unrestricted use, distribution, and reproduction in any medium, provided the original work is properly cited. of high-speed trains are made of thick metal materials, the direct communication link between users and base stations (eNodeB) will bare the penetration loss of 20-30 dB [4]. Usually, convergence antennas named onboard transceivers will be deployed on top of the carriage to play the role of mobile relay stations. Passengers will communicate through WiFi and Femtocell which exist in the carriage [5]. We also notice that, for the number of passengers on high-speed train is large and there is a growing demand on data traffic, multiple onboard transceivers can be deployed on the top of carriages as convergence point theoretically. On one hand, they can increase the chance to trigger the handover when the train goes through the overlapping region of neighboring cells, then the handover success probability can be improved by this means. On the other hand, the multiuser diversity or selection diversity [6] can be exploited to increase the system performance. That is, at any time, the system resource being allocated to the user with best channel condition will maximize the system throughput. Figure 1 illustrates the onboard transceivers on the top of carriages. To obtain multiuser diversity gain, the fluctuation of users' channels should have big 


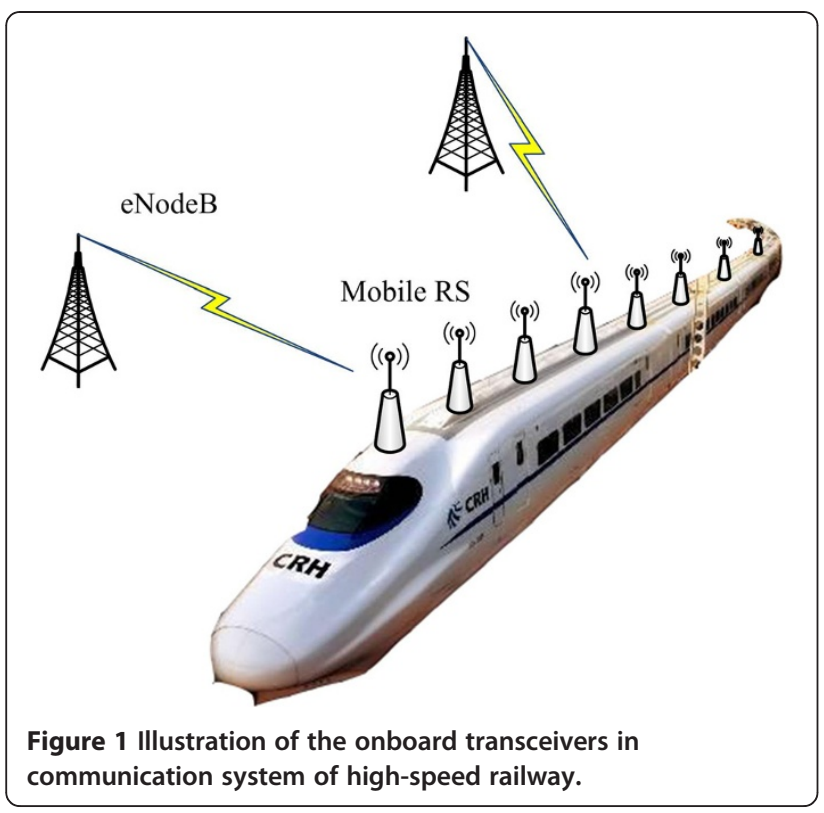

dynamic range. In the scenario of high-speed railway, the onboard transceivers usually have strong line-of-sight path to eNodeB except when the train goes through tunnels or mountainous areas. According to the analysis of [7] which states that little scattering results in small dynamic range of channel fluctuations, this is not good for multiuser diversity. To compensate for this deficiency, opportunistic beamforming (OBF), as one of the supporting technologies, is employed to change the dynamic range of channel magnitude by multiplying a complex weight on each antenna to make effective use of multiuser diversity. "Opportunistic" here means the probability that there exists a user whose instantaneous channel gains are close to matching the current powers and phases allocated at the transmit antennas. Beamforming implies that multiple antennas are used to form the transmission or reception beam and increase the signalto-noise ratio (SNR) at the receiver [8]. The sum capacity can be achieved if beamforming with dirty-paper coding is used at the eNodeB with perfect channel state information to transmit signals to multiple users $[9,10]$.

The common beamforming technique we refer to belongs to smart antenna techniques in which desired users' direction-of-arrival (DOA) is estimated from the channel condition feedback, then the direction of main lobe of the antenna is steered to the desired users [11]. By using this technique, the quality of received signal is improved without interfering undesired users. However, though the smart antennas can adjust the elevation, beam width, and azimuth angle, the changing is very slow [12]. Since the speed of the train is at least above $350 \mathrm{~km} / \mathrm{h}$ and the estimation error of DOA which are caused by feedback delay is large, beamforming technique of smart antennas cannot achieve expected effect. Instead, OBF is simply realized with dumb antennas by multiplied a complex vector of which the magnitude and phase both are random variables. Simultaneously, there is no need to track the channel vectors of users. Only SNR should be reported to eNodeBs and the user with highest SNR will be scheduled. When there are enough users in the system, multiuser diversity gain will be obtained. Certainly, the amount of OBF overhead is much smaller than that of smart antenna systems. However, compared with omni-directional antenna system, it costs more overhead and complexity to achieve better performance.

In the communication system of high-speed railway, location information is predictable. With the help of location information and length of the train, the range of DOAs of onboard transceivers can be calculated. This angle range can be used to make the generation of random complex weight vector more precise to extend the dynamic range of channel magnitude.

In this article, OBF algorithm is applied to the scenario of high-speed railway under the assumption of equal spacing onboard transceivers deployed on the top of carriages. Simulation results show that conventional OBF can bring some performance gain. With the help of location information, the performance can be improved further. So far as we know, no similar correlated study has been reported on the OBF for high-speed railway as well as its location information assisted version.

The rest of this article is organized as follows. Section 2 introduces the system and signal models of this article; Section 3 describes the principles of OBF and its application to high-speed railway simply; Section 4 analyzes how to improve OBF for high-speed railway with known location information; Section 5 contains simulation results and performance analysis; Section 6 concludes the article.

\section{Methods}

\section{System and signal model}

The communication system in high-speed railway has a linear topology as shown in Figure 2. The cell radius is $R$ and the vertical distance from eNodeB to the track is $d_{\text {min }}$. It is assumed that the eNodeB has $N_{t}$ antennas and each onboard transceiver has one single antenna. In this article, only the hop from eNodeB to onboard transceiver is considered [13]. The communication between passengers and onboard transceivers is beyond the scope of this study.

To obtain the multiuser diversity gain, it is assumed that onboard transceivers should be deployed as many as possible in the condition that the independence of received signals can be achieved. Assume that there is an onboard transceiver on the head of the train as well 


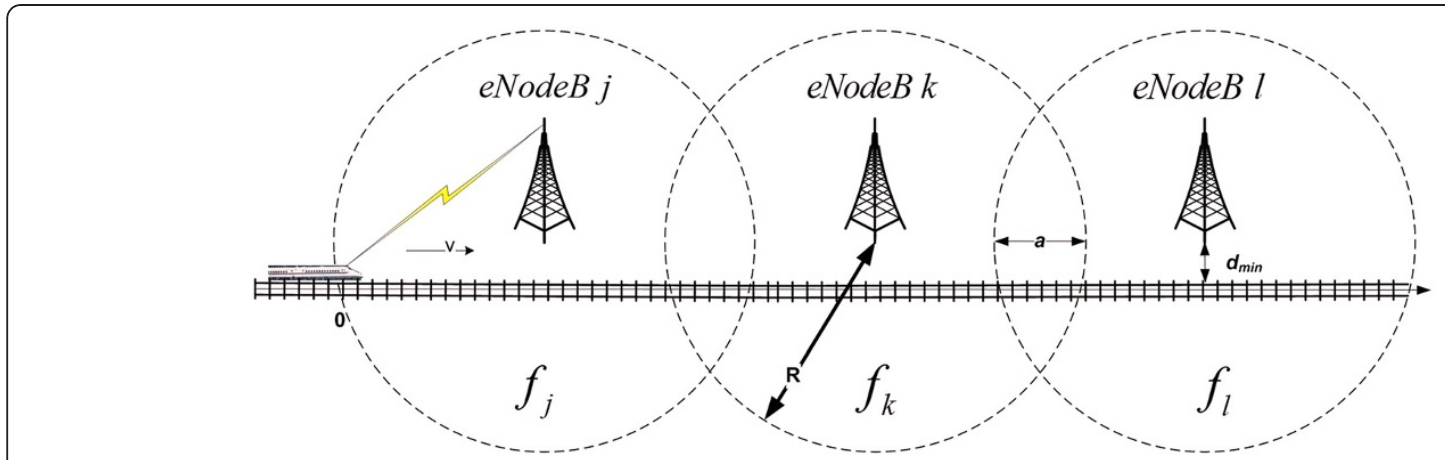

Figure 2 Linear network topology.

as on the tail and others are spacing equally distributed between the tail and head of the train. All of them are in a parallel line of the train. If there are $U$ transceivers and the length of the train is known as $L$, then the equal spacing will be $L /(U-1)$.

In this article, we assume that the transmit antennas are at close proximity, so the fading gains of the antennas are correlated [7]. Without loss of generality, we substitute "user" for "onboard transceiver" in the rest context. For user $m$, the correlated Rician channel between it and antenna $n$ can be expressed as [14]

$$
h_{n m}(t)=\sqrt{\frac{K_{m}}{1+K_{m}}} \exp \left(j \theta_{n m}\right)+\sqrt{\frac{1}{1+K_{m}}} b_{n m}(t)
$$

where $K_{m}$ denotes the Rician $K$-factor of the physical channel from the base station to user $m$. The angle is computed as follows [15]

$$
\theta_{n m}=\frac{2 \pi n d f_{c} \cos \Theta_{m}}{c}
$$

where spacing $d$ between two antenna elements is not larger than half a wavelength, $\Theta_{m}$ denotes the angle of the direct path to user k's moving direction or the DOA of user $k$ as shown in Figure 3 [16].

In Equation (1), the scattered component is modeled by $b_{n m}(t)$, which is an independent complex Gaussian random variable with zero mean and variance one: $b_{n m} \sim C N(0,1)$.

For high-speed railway, assume that speed and location information of the train control system is shared, then the DOA can be expressed as

where $d_{m}$ denotes the distance gone through by user $m$, and the time when the train first arrives at the cell edge is set as 0 .

$$
\Theta_{m}=\left\{\begin{array}{lr}
\operatorname{arccot}\left(\frac{\sqrt{R^{2}-d_{\min ^{2}}}-d_{m}}{d_{\min }}\right), & \text { for } 0 \leq d_{m} \leq \sqrt{R^{2}-d_{\min ^{2}}} \\
\pi-\operatorname{arccot}\left(\frac{\left.d_{m}-\sqrt{R^{2}-d_{\min ^{2}}}\right),}{d_{\min }}\right) & \text { for } \sqrt{R^{2}-d_{\min ^{2}}}<d_{m} \leq 2 \sqrt{R^{2}-d_{\min ^{2}}}
\end{array}\right.
$$

Principle and application of OBF in high-speed railway

Usually, eNodeBs need the users to feedback information to get the individual channel amplitude and phase from transmit antennas in order to beamform to a particular user. However, if there are many users in the system, when a user's overall channel SNR is near its peak, it will be scheduled a transmission with proper scheduling algorithm to achieve multiuser diversity. In this context, OBF is introduced [7]: by varying the powers and phases allocated to the transmit antennas, a beam is randomly swept and at any time transmission is scheduled to the user currently closest to the beam. With many users, there is likely to be a user very close to the beam at any time.

In conventional OBF [17], at time slot $t$, the same block of symbols $x(t)$ is multiplied by a complex number $\sqrt{\alpha_{n}(t)} \exp \left(j \phi_{n}(t)\right)$ at antenna $n$ and transmitted, respectively, from all the antennas, for $n=1, \ldots, N$, such that $\sum_{n=1}^{N} \alpha_{n}(t)=1$, preserving the total transmit power. The received signal model from all $N_{t}$ transmit antennas to user $m$ is given by

$$
y_{m}(t)=\left(\sum_{n=1}^{N_{t}} \sqrt{\alpha_{n}(t)} \exp \left(j \phi_{n}(t)\right) h_{n m}(t)\right) x(t)+\eta_{m}(t)
$$

where $\eta_{m}(t)$ is the additive white Gaussian noise with $\eta_{m}(t) \sim \mathrm{CN}\left(0, \sigma^{2} I_{T}\right)$. The overall channel gain for user $m$ is

$$
\tilde{h}_{m}(t)=\sum_{n=1}^{N_{t}} \sqrt{\alpha_{n}(t)} \exp \left(j \phi_{n}(t)\right) h_{n m}(t)
$$




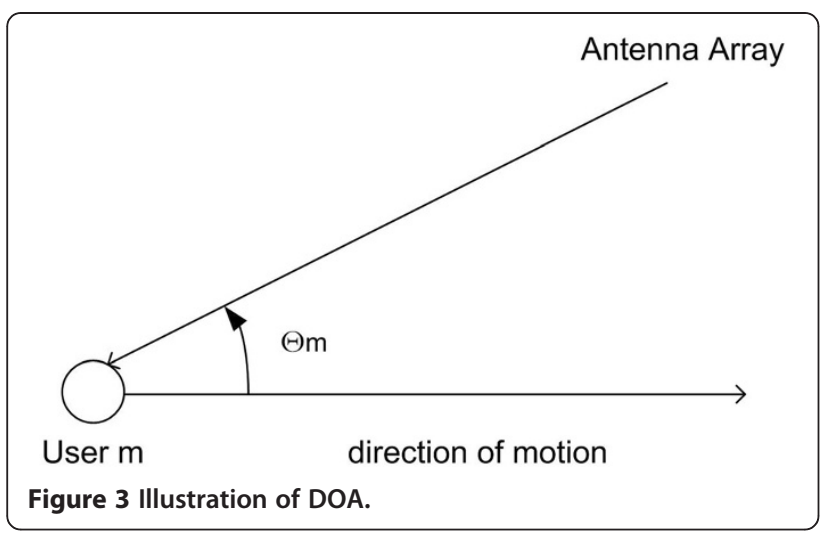

For correlated Rician channel, it can be further expressed as

Let $B_{m}(t)=\sqrt{\frac{1}{1+K_{m}}} \sum_{n=1}^{N_{t}} \sqrt{\alpha_{n}(t)} \exp \left(j \phi_{n}(t)\right) b_{n m}(t)$ and it is easy to prove that $B_{m}(t)$ is a complex-valued Gaussian random variable with $B_{k}(t) \sim C N\left(0, \sigma_{m}{ }^{2}\right)$, where $\sigma_{m}^{2}=\frac{1}{1+K_{m}}$.

Let $H_{m}(t)$ denote the magnitude of $\widetilde{h}_{m}(t)$ :

$$
\begin{aligned}
H_{m}(t) & =\left|\widetilde{h}_{m}(t)\right| \\
& =\left|\sqrt{\frac{K_{m}}{1+K_{m}}} \sum_{n=1}^{N_{t}} \sqrt{\alpha_{n}(t)} \exp \left(j\left(\phi_{n}(t)+\theta_{n m}\right)\right)+B_{m}(t)\right|
\end{aligned}
$$

According to [6], $\sqrt{\alpha_{n}(t)}=\frac{1}{\sqrt{N}}$ and combined with (2) to maximize the magnitude of overall channel, the phase can be generated as follows

$$
\phi_{n}(t)=-\frac{2 \pi n d f_{c} \cos \psi_{m}}{c}
$$

where $\psi_{m} \sim U(0,2 \pi)$.

\section{Location information-assisted OBF for high-speed railway} Communication systems of high-speed railway not only have kinds of disadvantages, but also have some inherent advantages. With the help of predictable location information, that is, at time slot $t$, the head of train arrives at location $s$, and the length of train is a constant, the location of the tail of the train can be got. Then, DOA of
Table 1 Simulation parameter

\begin{tabular}{ll}
\hline Parameter & Value \\
\hline Channel bandwidth $(\mathrm{BW})$ & $10 \mathrm{MHz}$ \\
\hline Antenna type & Dumb antennas \\
\hline Fixed directional antenna gain & $12 \mathrm{dBi}$ \\
\hline Beamforming antenna gain & $15 \mathrm{dBi}$ \\
\hline Number of transmit antennas & 4 \\
\hline Carrier frequency & $2 \mathrm{GHz}$ \\
\hline Rician factor $K$ & 6 \\
\hline eNodeB transmit power $\left(P_{\mathrm{eNB}}\right)$ & $43 \mathrm{dBm}$ \\
\hline eNodeB antenna height $\left(h_{b}\right)$ & $30 \mathrm{~m}$ \\
\hline Train antenna height $\left(h_{m}\right)$ & $1.5 \mathrm{~m}$ \\
\hline$d_{\text {min }}$ & $30 \mathrm{~m}$ \\
\hline Thermal noise density $\left(N_{0}\right)$ & $-174 \mathrm{dBm} / \mathrm{Hz}$ \\
\hline Cell radius $(R)$ & $3 \mathrm{~km}$ \\
\hline Site-to-site distance & $4.8 \mathrm{~km}$ \\
\hline Measurement periodic $\Delta t$ & $500 \mathrm{~ms}$ \\
\hline Speed & $360 \mathrm{~km} / \mathrm{h}$ \\
\hline
\end{tabular}

the users at the head and tail of the train can be calculated which are expressed as $\psi_{h}$ and $\psi_{t}$, respectively. If there are $U$ users and the length of the train is known as $L$, then the equal spacing will be $L /(U-1)$. For certain $U$, the distance between two neighboring RSs will increase with $L$. Higher $L$ also leads to larger angle range of $\left[\psi_{t}, \psi_{h}\right]$. Above all, for fixed number of RSs, the performance deteriorates with the increase of $L$.

In location information-assisted OBF scheme, the magnitude of the complex weight on each antenna is the same as in conventional one, but the phase is generated more precisely, that is, $\widetilde{\psi}_{m} \sim U\left(\psi_{t}, \psi_{h}\right)$. Then, the random phase can be expressed as

$$
\phi_{n}^{\mathrm{pro}}(t)=-\frac{2 \pi n d f_{c} \cos \tilde{\psi}_{m}}{c}
$$

For determinant Rician $K$-Factor, to maximize the magnitude of overall channel gain of user $m$ in Equation (7), $\phi_{n}(t)=-\theta_{n m}$ should be satisfied. At this time, the optimal beamforming is realized which is also called coherent beamforming. This is the upper bound of OBF performance.

$$
\begin{aligned}
\tilde{h}_{m}(t) & =\sum_{n=1}^{N_{t}} \sqrt{\alpha_{n}(t)} \exp \left(j \phi_{n}(t)\right) h_{n m}(t) \\
& =\sum_{n=1}^{N_{t}} \sqrt{\alpha_{n}(t)} \exp \left(j \phi_{n}(t)\right)\left(\sqrt{\frac{K_{m}}{1+K_{m}}} \exp \left(j \theta_{n m}\right)+\sqrt{\frac{1}{1+K_{m}}} b_{n m}(t)\right) \\
& =\sqrt{\frac{K_{k}}{1+K_{k}}} \sum_{n=1}^{N_{t}} \sqrt{\alpha_{n}(t)} \exp \left(j\left(\phi_{n}(t)+\theta_{n m}\right)\right)+\sqrt{\frac{1}{1+K_{m}}} \sum_{n=1}^{N_{t}} \sqrt{\alpha_{n}(t)} \exp \left(j \phi_{n}(t)\right) b_{n m}(t)
\end{aligned}
$$




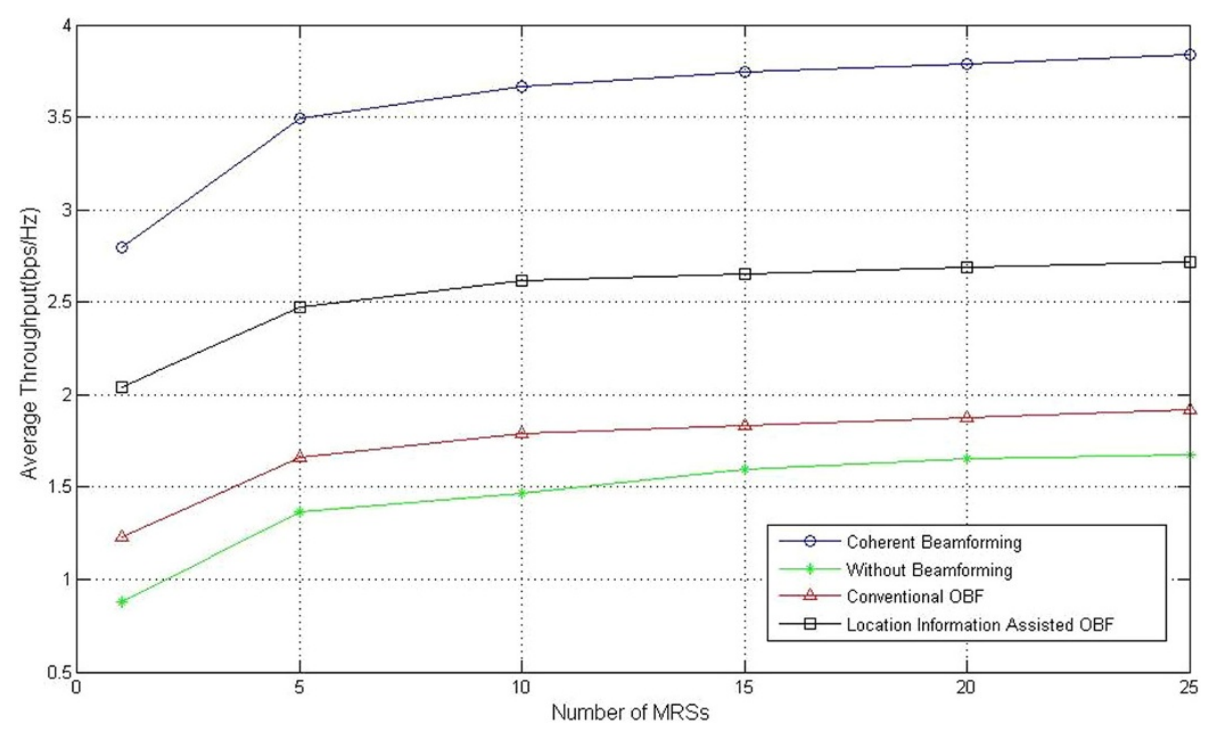

Figure 4 Impact of number of users on the average throughput.

According to Equations (2) and (8), in conventional OBF, we have

$$
\begin{aligned}
\phi_{n}(t)+\theta_{n m} & =-\frac{2 \pi n d f_{c} \cos \psi_{m}}{c}+\frac{2 \pi n d f_{c} \cos \Theta_{m}}{c} \\
& =\frac{2 \pi n d f_{c}}{c}\left(-\cos \psi_{m}+\cos \Theta_{m}\right)
\end{aligned}
$$

According to Equations (2) and (9), in location information assisted OBF, we have

$$
\begin{aligned}
\phi_{n}^{\mathrm{pro}}(t)+\theta_{n m} & =-\frac{2 \pi n d f_{c} \cos \tilde{\psi}_{m}}{c}+\frac{2 \pi n d f_{c} \cos \Theta_{m}}{c} \\
& =\frac{2 \pi n d f_{c}}{c}\left(-\cos \tilde{\psi}_{m}+\cos \Theta_{m}\right)
\end{aligned}
$$

Since $\psi_{m}{ }^{\sim} U(0,2 \pi)$ and $\tilde{\psi}_{m} \sim U\left(\psi_{t}, \psi_{h}\right)$, combined with Equation (3), there is a strong probability that the random phase generated by the proposed algorithm is much closer to $\Theta_{m}$. When the times of experiment are more enough, performance improvement can be observed.

\section{Simulation results and performance analysis}

The simulation results and analysis shown in this section are to verify that conventional OBF is proper for the scenario of high-speed railway and location information can assist this algorithm significantly. Detailed simulation parameters are shown in Table $1[18,19]$.

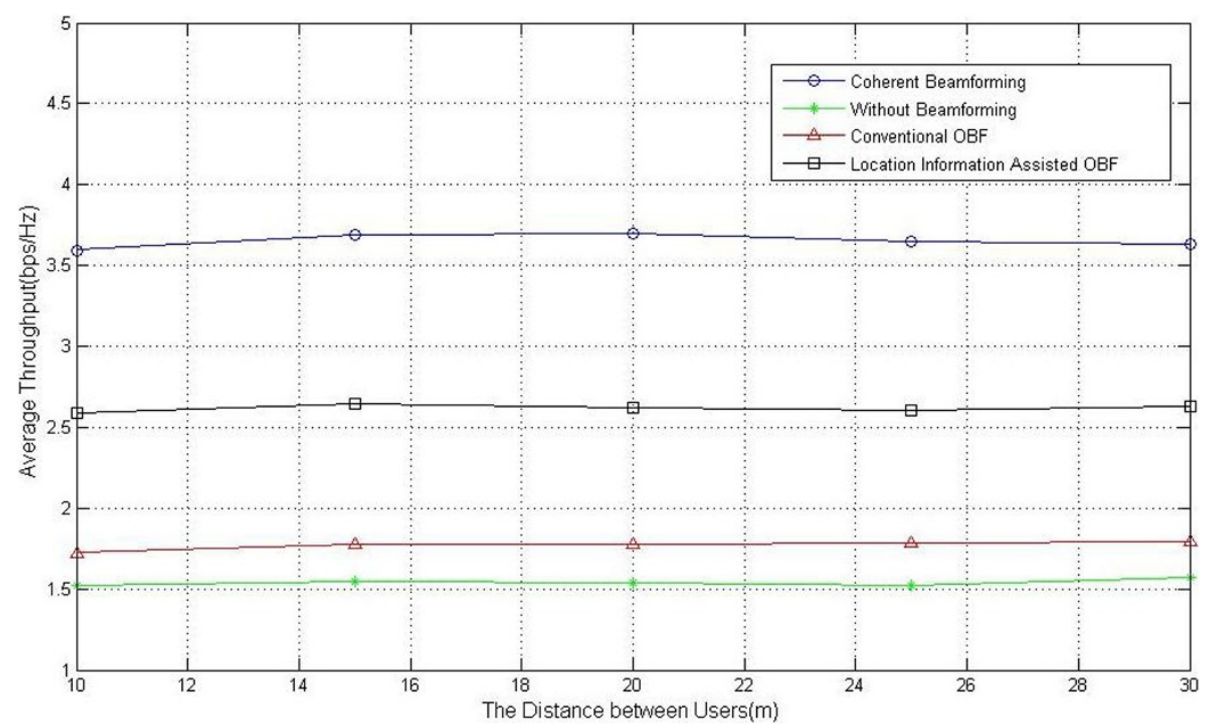

Figure 5 Impact of distance between users on the average throughput. In this simulation, the number of users is 11. 


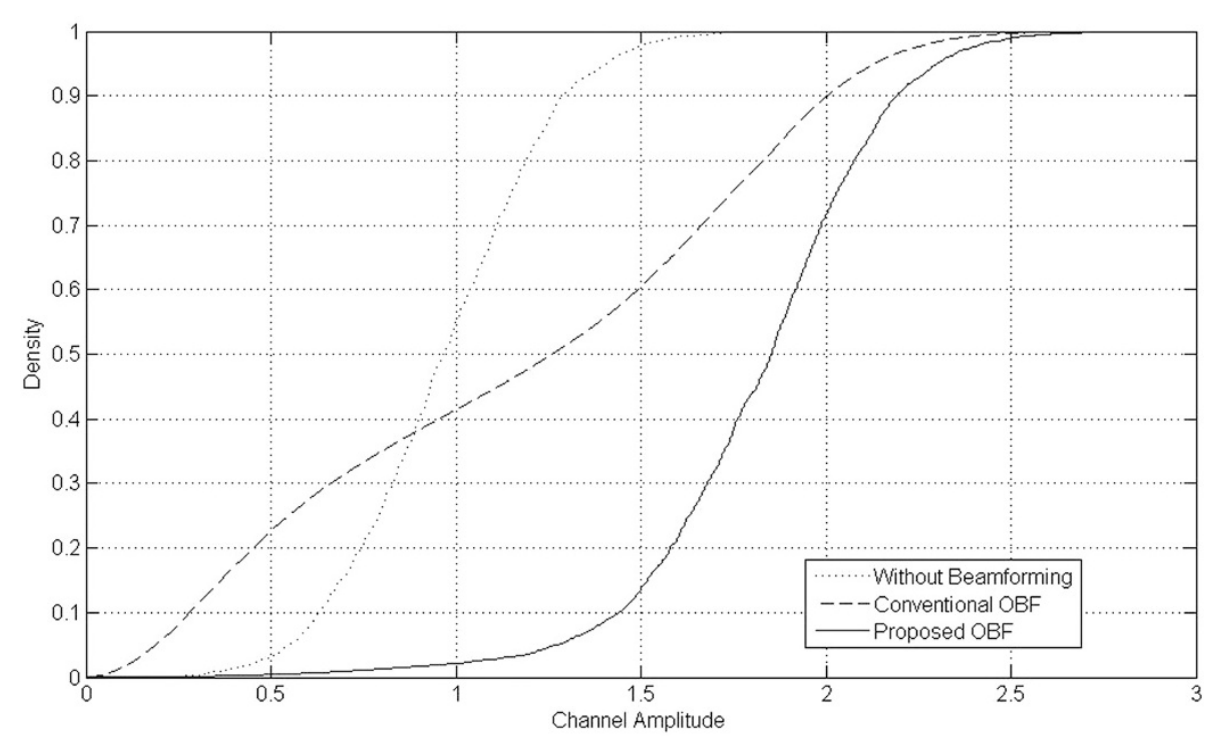

Figure 6 Impact of OBF on the distribution of channel amplitude.

In Figures 4 and 5, the line with circle denotes the performance of coherent beamforming [20]; the line with star denotes the performance without beamforming technique in which case the omni-directional antenna is employed; the lines with triangle and with square denote the performances of conventional OBF and location information-assisted $\mathrm{OBF}$, respectively. It is obvious that conventional OBF can bring performance gain to communication systems for high-speed railway and the inherent predictable location information can improve the performance further. However, as OBF is a kind of simple beamforming technique which can be realized by dumb antennas without real-time channel feedback, even with the help of location information, it still cannot perform as well as the optimal beamforming. The former one also shows that the average throughput increases with the number of users which is benefiting from multiuser diversity gain. The latter one of which the number of users is 11 illustrates that the distance between users has little influence on the performance if the independency of received signals can be guaranteed.

In Figure 6, we plot the distribution of the correlated Rician overall channel gains with conventional OBF and location information-assisted $\mathrm{OBF}$, and compare them to the case of omni-directional one. We can see how OBF influence the dynamic range of correlated Rician channel amplitudes. Conventional OBF can extend the range effectively. With the help of location information, the dynamic range is extended further. This is very useful to obtain multiuser diversity gain.

\section{Conclusions}

The characteristics of communication systems for highspeed railway have both pros and cons. For example, Doppler Effect and frequent handover are hindrances to good performance. The common beamforming technique of smart antennas cannot be normally employed due to the estimation error of DOA of desired users caused by channel feedback delay. On the contrary, OBF with dumb antennas which does not need the channel feedback of users is more proper for the scenario of high-speed railway. The inherent advantage such as regular movement and predictable location and speed information of this system can improve the conventional OBF and obtain multiuser diversity gain. However, current algorithm still cannot achieve or get very close to the performance upper bound set by coherent beamforming. In the future study, scheduling algorithm will be considered and beam selection will be added.

\section{Competing interests}

The authors declare that they have no competing interests.

\section{Acknowledgments}

The study was supported partially by the 973 Program under the Grant 2012CB316100, NSFC under the Grant 610711068, 61032002, and the Key Program of Technological R\&D of the Ministry of Railway under the Grant 2011X011-A.

Received: 15 February 2012 Accepted: 28 May 2012

Published: 3 July 2012

\section{References}

1. RD Pascoe, TN Eichorn, What is communication-based train control? IEEE Veh. Technol. Mag.4(4):16-21 (2009) 
2. UIC, LTE/SAE, The future railway mobile radio system? Long-term visions on railway mobile radio technologies. 4(2009). Draft

3. GP White, YV Zakharov, Data communications to trains from high-altitude platforms. IEEE Trans. Veh. Technol. 56(4):2253-2266 (2007)

4. F Abrishamkar, J Irvine, Comparison of current solutions for the provision of voice services to passengers on high speed trains, in Proc IEEE 52nd Veh Technol Conf (VTC'00 Fall), vol. 5 (Boston, MA, Boston, MA, 2000), pp. 2068-2075

5. LH Lu, XM Fang, M Cheng, CZ Yang, WT Luo, C Di, Positioning and relay assisted robust handover scheme for high speed railway, in Proc IEEE 73rd Veh Technol Conf (VTC'11 Spring), vol. 1 (Budapest, Hungary, 2011), pp. 1-5

6. R Knopp, P Humblet, Information capacity and power control in single cell multiuser communications, in Proc IEEE Int Computer Conf (ICC'95), vol. 1 (Seattle, WA, 1995), pp. 331-335

7. P Viswanath, DNC Tse, R Laroia, Opportunistic beamforming using dumb antennas. IEEE Trans. Inf. Theory 48,1277-1294 (2002)

8. E Dahlman, H Ekstrom, A Furuskar, Y Jading, J Karlsson, M Lundevall, S Parkvall, The $3 G$ long-term evolution radio interface concepts and performance evaluation, in IEEE 63rd Veh Technol Conf (VTC'06), vol. 1 (Melbourne, Australia, 2006), pp. 137-141

9. G Gaire, S Shamai, On the achievable throughput of a multiantenna Gaussian broadcast channel. IEEE Trans. Inf. Theory 49(7):1691-1706 (2003)

10. P Viswanath, D Tse, Sum capacity of the vector Gaussian broadcast channel and uplink-downlink duality. IEEE Trans. Inf. Theory 49(8):1912-1921 (2003)

11. CA Balanis, Antenna Theory (John Wiley, New Jersey, 2005)

12. $3 G$ Americas, $M I M O$ and smart antennas for $3 G$ and $4 G$ wireless systemspractical aspects and deployment considerations. (LTEWorld, 2007) (2011). http://teworld.org/whitepaper/mimo-and-smart-antennas-3g-and-4gwireless-systems-practical-aspects-and-deployment-consi

13. IST-4-027756 WINNER II, D1.1.2 v1.2. WINNER II Channel Models (2007) (2010). https://www.ist-winner.org/

14. IM Kim, ZH Yi, DW Kim, WS Chung, Improved opportunistic beamforming in Ricean channels. IEEE Trans. Commun. 54(12), 2199-2211 (2006). doi:10.1109/TCOMM.2006.884851

15. LC Godara, Application of antenna arrays to mobile communicationsPart II: beamforming and direction-of-arrival considerations. Proc. IEEE 85(8):1195-1245 (1997)

16. LC Godara, Smart Antennas (CRC Press, Florida, 2004)

17. DNC Tse, P Viswanath, Fundamentals of Wireless Communications (Cambridge University Press, Cambridge, 2005)

18. I Stojmenovic, Handbook of Wireless Networks and Mobile Computing (John Wiley, New York, 2001)

19. ITU-R, Guidelines for evaluation of radio interface technologies for IMTAdvanced (2011). http://www.itu.int/dms_pub/itu-r/opb/rep/R-REP-M.2135-12009-PDF-E.pdf

20. A Paulraj, R Nabar, D Gore, Introduction of Space-Time Wireless Communications (Cambridge University Press, Cambridge, 2003)

doi:10.1186/1687-1499-2012-210

Cite this article as: Cheng and Fang: Location information-assisted opportunistic beamforming in LTE system for high-speed railway. EURASIP Journal on Wireless Communications and Networking 2012 2012:210.

\section{Submit your manuscript to a SpringerOpen ${ }^{\odot}$ journal and benefit from:}

- Convenient online submission

- Rigorous peer review

- Immediate publication on acceptance

- Open access: articles freely available online

- High visibility within the field

- Retaining the copyright to your article

Submit your next manuscript at $\gg$ springeropen.com 